SNUST 02-0701

Short-Lectures at 2001 Les Houches Summer School

EXACT ANSWERS TO APPROXIMATE QUESTIONS

- NONCOMMUTATIVE DIPOLES, OPEN WILSON

LINES AND UV-IR DUALITY -

\author{
SOO-JONG REY \\ School of Physics \& Center for \\ Theoretical Physics, Seoul National \\ University, Seoul 151-747 KOREA
}




\section{Contents}

1 Introduction and Conjectures 3

2 Flying Noncommutative Dipole 5

3 Open Wilson Lines: How and Why?

3.1 Open Wilson Lines. .................... . . . 7

3.2 Generalized Star Products $\ldots \ldots \ldots \ldots$

\begin{tabular}{|lll}
4 & Free and Interacting OWLS & 11
\end{tabular}

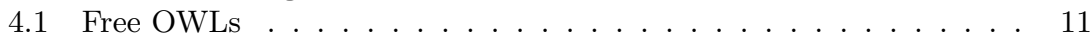

4.2 Interacting OWL $\ldots \ldots \ldots \ldots$

$\begin{array}{lll}5 \text { Closed Strings out of Open Strings } & 17\end{array}$

5.1 Open String as Miniature Dipole . . . . . . . . . . . . . . . . 17

5.2 Witten's $\star_{\mathrm{w}}$-product is Moyal's $\star_{\mathrm{m}}$-product . . . . . . . . . . . 18

5.3 Closed Strings as OWL $\ldots \ldots \ldots \ldots \ldots \ldots$ 


\title{
EXACT ANSWERS TO APPROXIMATE QUESTIONS - NONCOMMUTATIVE DIPOLES, OPEN WILSON LINES AND UV-IR DUALITY -
}

\author{
Soo-Jong Rey
}

\begin{abstract}
In this lecture, I put forward conjectures asserting that, in all noncommutative field theories, (1) open Wilson lines and their descendants constitute a complete set of interpolating operators of 'noncommutative dipoles', obeying dipole relation, (2) infrared dynamics of the noncommutative dipoles is dual to ultraviolet dynamics of the elementary noncommutative fields, and (3) open string field theory is a sort of noncommutative field theory, whose open Wilson lines are interpolating operators for closed strings. I substantiate these conjectures by various intuitive arguments and explicit computations of one- and two-loop Feynman diagrammatics.
\end{abstract}

\section{Introduction and Conjectures}

The most salient feature of noncommutative field theories, as opposed to the conventional commutative field theories, is that physical excitations are described by a 'noncommutative dipole' - weakly interacting, nonlocal object. One can visualize them as follows. Denote their center-of-mass momentum and dipole moment as $\mathbf{k}$ and $\ell$, respectively. According to the 'dipole' picture, originally developed in [1] and more recently reiterated in [2], the two quantities are related each other:

$$
\ell^{a}=\theta^{a b} \mathbf{k}_{b} .
$$

Here, $\theta^{a b}$ denotes the noncommutativity parameter 1 :

$$
\left\{\mathbf{x}^{a}, \mathbf{x}^{b}\right\}_{\star}=i \theta^{a b} .
$$

\footnotetext{
${ }^{1}$ Here, $\{\cdot\}$ refers to the Moyal commutator, defined in terms of the $\star$-product: $\left\{A\left(x_{1}\right) B\left(x_{2}\right)\right\}_{\star}:=\exp \left(\frac{i}{2} \partial_{1} \wedge \partial_{2}\right) A\left(x_{1}\right) B\left(x_{2}\right) \quad$ where $\quad \partial_{1} \wedge \partial_{2}:=\theta^{a b} \partial_{1}^{a} \partial_{2}^{b}$.
} 
Evidently, in the commutative limit, $\theta^{a b} \rightarrow 0$, the dipole shrinks in size and reduces to pointlike excitations.

Operationally, a noncommutative field theory is defined by an action functional of putative elementary fields, collectively denoted as $\Phi$. Elementary quanta are created/annihilated by none other than the elementary fields, $\Phi$. Apart from radiative corrections at higher-orders, in weakcoupling perturbation theory, they constitute a complete set of pointlike excitation spectrum. On the other hand, the above argument implies that, in a generic noncommutative field theory, in addition to point-like excitations, there ought to be dipole-like excitations as well. An immediate and interesting question is 'In what precise manner, does the noncommutative field theory produce dipole-like excitations?' . A related questions are 'If the dipole-like excitations are present, does that imply that the elementary field $\Phi$ is non-unitary? If so, should one introduce a new field for each dipole-like excitation?'. I claim that all these provoking questions can be answered, in noncommutative field theories, by introducing a set of operators, nicknamed as open Wilson lines (OWLs) 3, 3 ,

I found it illuminating to draw an analogy with the situation in QED. In QED, the spectrum includes, in addition to electron and proton quanta created/annihilated by respective fundamental fields, bound-states such as positronium or hydrogen atom, whose characteristic scale in wave functions or in 'parton distribution functions'is set by the Bohr radius. This does not mean, however, that perturbative unitarity is violated, or a new field creating/annihilating the positronium or the hydrogen atom needs to be introduced. Rather, the bound-states are properly understood as poles of two-particle-irreducible Green functions, for which the only technical difficulty would be non-analyticity of the fixed-order perturbation theory near the two-particle threshold. The perturbation theory remains valid outside the threshold region, and hence is better than the approach relying only on dispersion relation, for which the discontinuity across the branch cut ought to be known for all energies. Morally speaking, I view the noncommutative dipole as counterpart of the QED bound-state, created/annihilated by the open Wilson line operators.

In answering the question posed above, I put forward the following conjectures:

- 1. open Wilson lines: In a generic noncommutative field theory, there always exist a special class of composite operators, open Wilson lines, $W_{k}[\Phi]$, and their descendants, $(\Phi W)_{k}[\Phi]$.

- 2. noncommutative dipoles: The open Wilson lines and their descendants constitute a complete set of interpolating operators for creating/annihilating the 'noncommutative dipoles', obeying the dipole relation, Eq.(11.1).

- 3. UV-IR duality: Infrared dynamics of the noncommutative dipoles, and hence the open Wilson lines, $W_{k}[C]$, is dual to ultraviolet dynamics of the 
elementary fields, $\Phi$ 's.

- 4. Closed Strings from Open Strings: Extended to string field theories, open Wilson lines made out of open string field are interpolating operators for closed strings, both on and off-shell.

I would like to motivate these conjectures from the following considerations. Once one recalls the Weyl-Moyal correspondence, the conjecture 1 is rather transparent: the open Wilson lines in Moyal formulation correspond, in Weyl formulation, to the familiar Wilson loops, viz. 'master fields' in the large $N \sim \operatorname{Pf} \theta$ limit. The well-known fact that Wilson loops form a complete set of gauge-invariant operators in the large- $N$ gauge theory motivates the first half of the conjecture 2. That the open Wilson lines ought to obey the dipole relation, Eq.(1.1), will be proven in section 3. The conjecture 3 constitutes the most important feature regarding the long-distance spectrum and dynamics of generic noncommutative field theories. Evidently, the conjectured duality is strongly reminiscent of the 'channel' duality between open and closed strings, and implies that the open Wilson lines are operators associated with 'closed' string-like excitations in noncommutative field theories. The conjecture 4 asserts that the open string field theory is a sort of noncommutative field theory and that closed strings are describable entirely in terms of the open Wilson lines made out of open string field.

In this lecture, I take the simplest yet interacting noncommutative field theory, $d$-dimensional $\lambda\left[\Phi^{3}\right]_{\star}$ theory, and substantiate the above conjectures 1 - 4. Specifically, I present relevant results from computation of one- and two-loop effective action. I prove explicitly that the effective action is expressible entirely in terms of scalar open Wilson lines, and that interaction among the open Wilson lines is noncommutative and purely geometrical. Viewing the theory as level-zero truncation of the Witten's open string field theory, I also argue that the scalar open Wilson lines act precisely as the closed string field.

\section{Flying Noncommutative Dipole}

I begin with a physical situation from which intuitive picture of the noncommutative dipole is developable: Mott exciton in a strong magnetic field. As is well-known, in a strong magnetic field, low-energy excitation of electrons and holes is projected to the lowest Landau level so that, in the quasiparticle's Hamiltonian, the kinetic energy is negligible compared to the residual potential energy such as Coulomb interaction energy. An immediate question is whether the situation repeats for charge-neutral excitons, viz. bound-state consisting of an equal number of electrons and holes. Following the pioneering works [1], I now prove that low-energy excitation of the Mott exciton consists only of rigid translational motion in the plane perpendicular to the magnetic field. 
In the non-relativistic limit, the Hamiltonian of a charge-neutral exciton in the background of a uniform electric and magnetic field, $\mathbf{E}$ and $\mathbf{B}$, is given by

$H=\frac{\left(\mathbf{p}_{1}+e \mathbf{A}\left(\mathbf{r}_{1}\right)\right)^{2}}{2 m}+\frac{\left(\mathbf{p}_{2}-e \mathbf{A}\left(\mathbf{r}_{2}\right)\right)^{2}}{2 m}+e \mathbf{E} \cdot\left(\mathbf{r}_{1}-\mathbf{r}_{2}\right)+V\left(\left|\mathbf{r}_{1}-\mathbf{r}_{2}\right|\right)$.

The velocity operator of the electron and the hole is given by $\mathbf{v}_{1,2}=\partial H$ / $\partial \mathbf{p}_{1,2}$, and obeys the operator equations of motion:

$$
m \frac{d \mathbf{v}_{1}}{d t}=-e \mathbf{E}+e \mathbf{B} \wedge \mathbf{v}_{1} \quad \text { and } \quad m \frac{d \mathbf{v}_{2}}{d t}=+e \mathbf{E}-e \mathbf{B} \wedge \mathbf{v}_{2} .
$$

One readily finds that the total momentum of the excition, $\mathbf{P}:=m\left(\mathbf{v}_{1}+\right.$ $\left.\mathbf{v}_{2}\right)-e \mathbf{B} \wedge\left(\mathbf{r}_{1}-\mathbf{r}_{2}\right)$ is conserved: $d \mathbf{P} / d t=[H, \mathbf{P}]=0$. Take the symmetric gauge $\mathbf{A}(\mathbf{r})=\frac{1}{2} \mathbf{B} \wedge \mathbf{r}$. The conserved total momentum is then given by

$$
\mathbf{P}=\left(\mathbf{p}_{1}+\mathbf{p}_{2}\right)-\frac{e}{2} \mathbf{B} \wedge\left(\mathbf{r}_{1}-\mathbf{r}_{2}\right)
$$

In terms of the center-of-mass coordinate, $\mathbf{R}=\left(\mathbf{r}_{1}+\mathbf{r}_{2}\right) / 2$ and the relative coordinate, $\ell:=\left(\mathbf{r}_{1}-\mathbf{r}_{2}\right)$, the exciton wave-function is given by

$$
\Psi_{\mathbf{P}}\left(\mathbf{r}_{1}, \mathbf{r}_{2}\right)=\exp \left(i \mathbf{R} \cdot \mathbf{P}+\frac{i e}{2} \mathbf{R} \wedge \Delta \mathbf{x}\right) \psi_{\mathrm{P}}(\ell)
$$

and, after straightforward algebra, one obtains the total Hamiltonian as

$$
H=\frac{1}{B^{2}} \mathbf{P} \cdot \mathbf{E} \wedge \mathbf{B}-\frac{1}{2} M \frac{\mathbf{E}^{2}}{\mathbf{B}^{2}}+H_{\mathrm{rel}}, \quad(M=2 m)
$$

To make contact with noncommutative field theories more transparent, introduce noncommutativity parameter $\theta^{a b}=\left(\mathbf{F}^{-1}\right)^{a b}$, and inverse metric $G^{a b}=\left(-\theta^{2}\right)^{a b}$. One then readily derives the operator relations for the exciton center-of-mass velocity $\mathbf{V}$ :

$$
\mathbf{V}^{a}=\frac{\partial H}{\partial \mathbf{P}}=\theta^{a b} \mathbf{E}_{b}
$$

and for the exciton electric dipole moment $\mathbf{d} \equiv e \ell$ :

$$
\mathbf{d}^{a} \equiv \frac{\partial H}{\partial \mathbf{E}_{a}}=M G^{a b} \mathbf{E}_{b}+\theta^{a b} \mathbf{P}_{b}
$$

These are precisely the noncommutative dipole relation, Eq.(1.1).

Moreover, the relative Hamiltonian $H_{\text {rel }}$ turns out identical with the standard Landau-level problem for a charged particle (with reduced mass). 
Thus, in the strong magnetic field limit, approximating the lowest Landau level wavefunction by Dirac delta-function, one obtains the excitation wavefunction as:

$$
\Psi_{\mathbf{P}}(\mathbf{R}, \mathbf{r}) \sim \exp \left(i \mathbf{R} \cdot \mathbf{P}+\frac{i e}{2} \mathbf{R} \wedge \ell\right) \delta(\mathbf{r}-\ell) .
$$

The wave-function Eq.(2.1) proves that the low-energy dynamics of the Mott exciton comprises of rigid translation, whose dipole moment is proportional to the the center-of-mass momentum.

\section{Open Wilson Lines: How and Why?}

\subsection{Open Wilson Lines}

How, in a given noncommutative field theory, are the open Wilson lines defined, and what are they for? I claim that the answer lies ultimately to the observation alluded above: noncommutative dipoles are present generically as part of theory's low-energy excitation. In noncommutative gauge theories, the answer also has to do with gauge-invariant, physical observables, so I will begin with this case first. In [3 5], it has been shown that (part of) gauge orbit is equivalent to the translation along the noncommutative directions. For example, in noncommutative U(1) gauge theory, the gauge potential $\mathbf{A}_{\mu}(x)$ and the neutral scalar field $\Phi(x)$, both of which give rise to 'noncommutative dipoles', transform in 'adjoint' representation:

$$
\begin{aligned}
\delta_{\epsilon} \mathbf{A}_{\mu}(x) & =i \int \frac{\mathrm{d}^{2} \mathbf{k}}{(2 \pi)^{2}} \widetilde{\epsilon}(\mathbf{k})\left[\left(\mathbf{A}_{\mu}(\mathbf{x}+\theta \cdot \mathbf{k})-\mathbf{A}_{\mu}(\mathbf{x}-\theta \cdot \mathbf{k})\right)+i \mathbf{k}_{\mu}\right] e^{i \mathbf{k} \cdot \mathbf{x}} \\
\delta_{\epsilon} \Phi(x) & =i \int \frac{\mathrm{d}^{2} \mathbf{k}}{(2 \pi)^{2}} \widetilde{\epsilon}(\mathbf{k})[\Phi(\mathbf{x}+\theta \cdot \mathbf{k})-\Phi(\mathbf{x}-\theta \cdot \mathbf{k})] e^{i \mathbf{k} \cdot \mathbf{x}}
\end{aligned}
$$

where the infinitesimal gauge transformation parameter is denoted as

$$
\epsilon(\mathbf{x})=\int \frac{\mathrm{d}^{2} \mathbf{k}}{(2 \pi)^{2}} e^{i \mathbf{k} \cdot \mathbf{x}} \widetilde{\epsilon}(\mathbf{k}) .
$$

Because of the peculiarity, for fields transforming in 'adjoint' representations under the noncommutative gauge group, there is no physical observables local in configuration space. The only physical observable one can construct turn out local in momentum space, and is referred as the 'open Wilson line' operators [3 6] and their descendants defined on an open contour $C$ :

$$
\begin{aligned}
W_{\mathbf{k}}[\mathbf{A}] & =\mathcal{P}_{\mathrm{t}} \int \mathrm{d}^{2} \mathbf{x} \exp _{\star}\left(i \int_{0}^{1} \mathrm{~d} t \dot{\mathbf{y}} \cdot \mathbf{A}(x+\mathbf{y})\right) \star e^{i \mathbf{k} \cdot \mathbf{x}} \\
(\mathcal{O} W)_{\mathbf{k}}[\mathbf{A}] & =\mathcal{P}_{\mathrm{t}} \int \mathrm{d}^{2} \mathbf{x}\left[\int_{0}^{1} \mathrm{~d} t \mathcal{O}(t) \exp _{\star}\left(i \int_{0}^{1} \mathrm{~d} t \dot{\mathbf{y}} \cdot \mathbf{A}(x+\mathbf{y})\right)\right] \star e^{i \mathbf{k} \cdot \mathbf{x}} .
\end{aligned}
$$


Here, the $\star$-product is defined with respect to the base point $\mathbf{x}$ of the open contour $C$. Despite being defined over an open contour, the operator is gauge-invariant provided the momentum $\mathbf{k}$ is related to the geodesic distance $\mathbf{y}(1)-\mathbf{y}(0):=\Delta \mathbf{x}$ precisely by the 'dipole relation', Eq.(1.1). In other words, in noncommutative gauge theory, the open Wilson lines (physical observables) are noncommutative dipoles, obeying the dipole relation Eq.(1.1) as an immediate consequence of the gauge invariance!

The open Wilson lines are actually ubiquitous and are present in generic noncommutative field theories, in which neither gauge invariance nor gauge field is present. This is because, as I have convinced you already, the dipole relation Eq.(1.1) ought to be a universal relation, applicable for any theories defined over noncommuative spacetime. In [7 9, I have shown that the scalar open Wilson line operators $W_{\mathbf{k}}[\Phi]$ and descendants $\left(\Phi^{n} W\right)_{\mathbf{k}}[\Phi]$ are given by:

$$
\begin{aligned}
W_{\mathbf{k}}[\Phi] & :=\mathcal{P}_{\mathrm{t}} \int \mathrm{d}^{2} \mathbf{x} \exp \left(i g \int_{0}^{1} \mathrm{~d} t|\dot{\mathbf{y}}(t)| \Phi(x+\mathbf{y}(t))\right) \star e^{i \mathbf{k} \cdot \mathbf{x}} \\
\left(\Phi^{n} W\right)_{\mathbf{k}}[\Phi] & :=\left(-i \frac{\partial}{\partial g}\right)^{n} W_{\mathbf{k}}[\Phi] \quad(n=1,2,3, \cdots),
\end{aligned}
$$

where $\lambda$ is an appropriate coupling parameter.

Can one show that the dipole relation Eq. 1.1. is satisfied for scalar fields, wherein no gauge invariance is present? Consider the following set of operators, so-called Parisi operators [10]:

$\mathcal{O}_{n}\left(x_{1}, \cdots, x_{n} ; \mathbf{k}\right)=\int d^{2} \mathbf{z} \Phi_{1}\left(x_{1}+\mathbf{z}\right) \star \Phi_{2}\left(x_{2}+\mathbf{z}\right) \star \cdots \star \Phi_{n}\left(x_{n}+\mathbf{z}\right) \star e^{i \mathbf{k} \cdot \mathbf{x}}$,

viz. Fourier-transform of a string of elementary scalar fields, $\Phi_{k}(x)(k=$ $1,2, \cdots)$. Take the one-point function:

$$
G_{1}(x, \mathbf{k}):=\left\langle\mathcal{O}_{2}(x, \mathbf{k})\right\rangle=\left\langle\int d^{2} \mathbf{z} \Phi(\mathbf{z}) \star \Phi(x+\mathbf{z}) \star e^{i \mathbf{k} \cdot \mathbf{x}}\right\rangle .
$$

In terms of Fourier decomposition of the scalar field:

$$
\Phi(x)=\int \frac{d^{2} \mathbf{k}}{(2 \pi)^{2}} e^{i \mathbf{k} \cdot \mathbf{x}} \widetilde{\Phi}(\mathbf{k}),
$$

I obtain that

$$
G_{1}(x, \mathbf{k})=\int \frac{d^{2} \mathbf{l}}{(2 \pi)^{2}} \widetilde{\Phi}(\mathbf{l}) \widetilde{\Phi}(-\mathbf{l}+\mathbf{k}) \exp \left[i \mathbf{l} \cdot\left(x+\frac{1}{2} \theta \cdot \mathbf{k}\right)\right] .
$$

Consider 'wave-packet' of the scalar particle, $\Phi(\mathbf{z})=\Phi_{0} \delta^{(2)}(\mathbf{z})$ and $\Phi(x+$ $\mathbf{z})=\Phi_{0} \delta^{(2)}(x+\mathbf{z})$ so that $\widetilde{\Phi}(\mathbf{l})=\Phi_{0} \exp (i \mathbf{l} \cdot x)$. From the above equation, 
I then find that

$$
G_{1}(x, \mathbf{k})=\Phi_{0}^{2} \delta^{(2)}\left(x+\frac{1}{2} \theta \cdot \mathbf{k}\right) .
$$

Thus, I find that the stationary point of the correlator is given by $\Delta x^{a} \sim$ $\theta^{a b} \mathbf{k}_{b}$, and hence precisely by the 'dipole relation', Eq.(1.1).

I also claim that the open Wilson lines are a sort of 'master fields'. According to the Weyl-Moyal correspondence, generic noncommutative fields, be they the gauge field $\mathbf{A}$ or the scalar field $\Phi$, are interpretable as $(N \times N)$ matrix-valued fields at $N \rightarrow \infty$ limit, living only on commutative directions (if there is any). Hence, from the latter formulation, one can construct Wilson loop operators as the large- $N$ master fields:

$$
W_{\mathbf{k}}[\widehat{A}]=\operatorname{Tr} \exp (i \mathbf{k} \cdot \widehat{\mathbf{A}}) \quad \text { or } \quad W_{k}[\widehat{\Phi}]=\operatorname{Tr} \exp (i k \widehat{\Phi}) .
$$

In fact, one can readily show that, once expanded around the noncommutative space, these Wilson loop operators turn into the aforementioned open Wilson lines.

\subsection{Generalized Star Products}

Computationally, the open Wilson lines originate from resummation of socalled generalized $\star$-products. As such, I will first indicate how the generalized $\star$-products are inherent to the defintion of the open Wilson lines.

Begin with the gauge open Wilson lines. For a straight contour, expanding Eq.(3.2) in successive powers of the gauge field $\mathbf{A}_{\mu}$, it was observed 11, 12 that generalized $\star$-product, $\star_{n}$, a structure discovered first in [13, 14], emerge:

$$
W_{\mathbf{k}}[C]=\int \mathrm{d}^{2} \mathbf{x}\left[1-(\partial \wedge \mathbf{A})+\frac{1}{2 !}(\partial \wedge \mathbf{A})_{\star_{2}}^{2}+\cdots\right] \star e^{i \mathbf{k} \cdot \mathbf{x}} .
$$

The generalized $\star_{n}$ product exhibits different algebraic structures from Moyal's $\star$-product. For instance, the first two, $\star_{2}, \star_{3}$ defined as

$$
\begin{aligned}
{\left[A\left(x_{1}\right) B\left(x_{2}\right)\right]_{\star_{2}}:=} & \frac{\sin \left(\frac{1}{2} \partial_{1} \wedge \partial_{2}\right)}{\frac{1}{2} \partial_{1} \wedge \partial_{2}} A\left(x_{1}\right) B\left(x_{2}\right) \\
{\left[A\left(x_{1}\right) B\left(x_{2}\right) C\left(x_{3}\right)\right]_{\star_{3}}:=} & {\left[\frac{\sin \left(\frac{1}{2} \partial_{2} \wedge \partial_{3}\right)}{\frac{1}{2}\left(\partial_{1}+\partial_{2}\right) \wedge \partial_{3}} \frac{\sin \left(\frac{1}{2} \partial_{1} \wedge\left(\partial_{2}+\partial_{3}\right)\right)}{\frac{1}{2} \partial_{1} \wedge\left(\partial_{2}+\partial_{3}\right)}+(1 \leftrightarrow 2)\right] } \\
& \times A\left(x_{1}\right) B\left(x_{2}\right) C\left(x_{3}\right)
\end{aligned}
$$

show that the $\star_{n}$ 's are commutative but non-associative. Despite these distinguishing features, I interpret Moyal's $\star$ product more fundamental 
than the generalized $\star_{n}$ products. The open Wilson line is defined in terms of path-ordered $\star$-product, and its expansion in powers of the gauge potential involves the generalized $\star_{n}$-product at each $n$-th order. As such, complicated $\star_{n}$ products arise upon expansion in powers of the gauge potential, and are attributible to dipole nature of the open Wilson line and the gauge invariance therein - each term in Eq.(3.4) is not gauge invariant, as the gauge transformation Eq.(3.1) mixes terms involving different $\star_{n}$ 's. Indeed, the generalized $\star_{n}$ products are not arbitrary but obey recursive identities:

$$
\begin{aligned}
i\left[\partial_{x} A \wedge \partial_{x} B\right]_{\star_{2}} & =\{A, B\}_{\star} \\
i \partial_{x} \wedge\left[A B \partial_{x} C\right]_{\star_{3}} & =A \star_{2}\{B, C\}_{\star}+B \star_{2}\{A, C\}_{\star} .
\end{aligned}
$$

These identities are crucial for ensuring gauge invariance of the power-series expanded open Wilson line operator, Eq.(3.4).

I can show readily that the same sort of generalized $\star$-products also show up in the scalar open Wilson lines. Consider a simplified form of the scalar open Wilson line with an insertion of a local operator $\mathcal{O}$ at a location $\mathbf{R}$ on the Wilson line contour:

$$
\left(\mathcal{O}_{\mathbf{R}} W\right)_{\mathbf{k}}[\Phi]:=\mathcal{P}_{\mathrm{t}} \int \mathrm{d}^{2} \mathbf{x} \mathcal{O}(x+\mathbf{R}) \star \exp \left(i g \int_{0}^{1} \mathrm{~d} t|\dot{\mathbf{y}}(t)| \Phi(x+\mathbf{y}(t))\right) \star e^{i \mathbf{k} \cdot \mathbf{x}} .
$$

Take, for simplicity, a straight Wilson line:

$$
\mathbf{y}(t)=\mathbf{L} t \quad \text { where } \quad \mathbf{L}^{a}=\theta^{a b} \mathbf{k}_{b}:=(\theta \cdot \mathbf{k})^{a}, \quad L:=|\mathbf{L}|,
$$

corresponding to a uniform distribution of the momentum $\mathbf{k}$ along the Wilson line. As the path-ordering progresses to the right with increasing $t$, power-series expansion in $g L \Phi$ yields:

$$
\begin{aligned}
\left(\mathcal{O}_{\mathbf{R}} W\right)_{\mathbf{k}}[\Phi] & =\int \mathrm{d}^{2} \mathbf{x} e^{i \mathbf{k} \cdot \mathbf{x}} \star[\mathcal{O}(\mathbf{x}+\mathbf{R}) \\
& +i g L \int_{0}^{1} \mathrm{~d} t \mathcal{O}(x+\mathbf{R}) \star \Phi(x+\mathbf{L} t) \\
& +(i g L)^{2} \int_{0}^{1} \mathrm{~d} t_{1} \int_{t_{1}}^{1} \mathrm{~d} t_{2} \mathcal{O}(\mathbf{x}+\mathbf{R}) \star \Phi\left(x+\mathbf{L} t_{1}\right) \star \Phi\left(x+\mathbf{L} t_{2}\right) \\
& +\cdots \quad] .
\end{aligned}
$$

I can evaluate each term, for instance, by Fourier-transforming $\mathcal{O}$ and $\Phi$ 's:

$\mathcal{O}(x)=\int \frac{\mathrm{d}^{2} \mathbf{k}}{(2 \pi)^{2}} \widetilde{O}(\mathbf{k}) T_{\mathbf{k}}, \quad \Phi(x)=\int \frac{\mathrm{d}^{2} \mathbf{k}}{(2 \pi)^{2}} \widetilde{\Phi}(\mathbf{k}) T_{\mathbf{k}} \quad$ where $\quad T_{\mathbf{k}}=e^{i \mathbf{k} \cdot \mathbf{x}}$, 
taking the $\star$-products of the translation generators: $T_{\mathbf{k}} \star T_{\mathbf{l}}=e^{\frac{i}{2} \mathbf{k} \wedge \mathbf{l}} T_{\mathbf{k}+\mathbf{l}}$, and then evaluating the parametric $t_{1}, t_{2}, \cdots$ integrals. Fourier-transforming back to the configuration space, after straightforward calculations, I obtain

$$
\begin{aligned}
\left(\mathcal{O}_{\mathbf{R}} W\right)_{\mathbf{k}}[\Phi] & =\int \mathrm{d}^{2} \mathbf{x} e^{i \mathbf{k} \cdot \mathbf{x}} \mathcal{O}(x+\mathbf{R}) \\
& +i g L \int \mathrm{d}^{2} \mathbf{x} e^{i \mathbf{k} \cdot \mathbf{x}}[\mathcal{O}(x+\mathbf{R}) \Phi(x)]_{\star_{2}} \\
& +\frac{1}{2 !}(i g L)^{2} \int \mathrm{d}^{2} \mathbf{x} e^{i \mathbf{k} \cdot \mathbf{x}}[\mathcal{O}(x+\mathbf{R}) \Phi(x) \Phi(x)]_{\star_{3}}+\cdots
\end{aligned}
$$

I then observe that the products involved, $\star_{2}, \star_{3}, \cdots$, are precisely the same generalized $\star_{n}$ products as those appeared prominently in the gauge open Wilson line operators.

\section{$4 \quad$ Free and Interacting OWLs}

To identify degrees of freedom associated with the open Wilson lines and to understand their spectrum and interaction, consider the $\lambda\left[\Phi^{3}\right]_{\star}$-theory, and study the effective action. The noncommutative Feynman rules of $\lambda\left[\Phi^{3}\right]_{\star^{-}}$ theory are summarized, in the background field method, by the following generating functional:

$$
Z\left[\Phi_{0}\right]=Z_{0}\left[\Phi_{0}\right] \int \mathcal{D} \varphi \exp \left(-\int \mathrm{d}^{d} x\left[\frac{1}{2} \varphi(x) \mathcal{D}_{\Phi_{0}} \varphi(x)+\frac{\lambda}{3 !} \varphi^{3}(x)\right]_{\star}\right) .
$$

Here, $\mathcal{D}_{\Phi_{0}}=\left(-\partial_{x}^{2}+m^{2}+\lambda \Phi_{0}(x)\right)$, and $\Phi_{0}(x)$ and $\varphi(x)$ refer to the background and the fluctuation parts of the scalar field, $\Phi$, respectively. Because of the noncommutativity of the $\star$-product, interactions are classifiable into planar and nonplanar ones. I focus on so-called nonplanar part of the oneand two-loop Feynman diagrams, and, as I am interested primarily in dynamics at long distance, on the low-energy and large noncommutativity limit:

$$
\frac{p}{m}=\mathcal{O}\left(\epsilon^{+1}\right), \quad m^{2} \theta^{a b}=\mathcal{O}\left(\epsilon^{-2}\right), \quad \frac{\lambda \Phi}{m^{2}}=\mathcal{O}\left(\epsilon^{+1}\right) \quad \text { as } \quad \epsilon \rightarrow 0^{+} .
$$

\subsection{Free OWLs}

Begin with effective action at one loop. The nonplanar part of the oneparticle-irreducible N-point Green function is given by (see Fig. 1)

$$
\begin{aligned}
\Gamma_{\mathrm{N}}\left(\left\{p_{i}\right\},\left\{q_{j}\right\}\right)= & \hbar\left(-\frac{\lambda}{2}\right)^{\mathrm{N}} \sum_{\mathrm{N}_{1}+\mathrm{N}_{2}=\mathrm{N}} \frac{C_{\{N\}}}{(4 \pi)^{d / 2}} \int_{0}^{\infty} \frac{\mathrm{dT}}{\mathrm{T}} \mathrm{T}^{-\frac{d}{2}+\mathrm{N}} \\
& \times \exp \left[-m^{2} \mathrm{~T}-\frac{\ell^{2}}{4 \mathrm{~T}}\right] J_{\mathrm{N}_{1}}(\ell) J_{\mathrm{N}_{2}}(-\ell) .
\end{aligned}
$$


Here, I have denoted an N-dependent combinatoric factor as $C_{\{\mathrm{N}\}}$, divided $\mathrm{N}$ external momenta into two groups: $\left\{p_{1}, \cdots p_{\mathrm{N}_{1}}\right\}$ and $\left\{q_{1}, \cdots, q_{\mathrm{N}_{2}}\right\}$, and defined $k=\sum_{i=1}^{\mathrm{N}_{1}} p_{i}=-\sum_{i=1}^{\mathrm{N}_{2}} q_{i}$, and $\ell:=\theta \cdot k$ (consistent with the noncommutative dipole relation). I have also defined $J_{\mathrm{N}}(\ell)$ by

$J_{\mathrm{N}_{1}}\left(\ell,\left\{p_{i}\right\}\right):=\int_{-1 / 2}^{1 / 2} \mathrm{~d} \tau_{1} \cdots \mathrm{d} \tau_{\mathrm{N}} \exp \left[-i \sum_{i=1}^{\mathrm{N}_{1}} \tau_{i} p_{i} \cdot \ell-\frac{i}{2} \sum_{i<j=1}^{\mathrm{N}_{1}} \epsilon\left(\tau_{i j}\right) p_{i} \wedge p_{j}\right]$,

where $\tau_{i j} \equiv\left(\tau_{i}-\tau_{j}\right)$, and similarly $J_{\mathrm{N}_{2}}\left(-\ell,\left\{q_{j}\right\}\right)$. They are precisely the momentum-space kernel of the generalized $*_{\mathrm{N}}$ product.

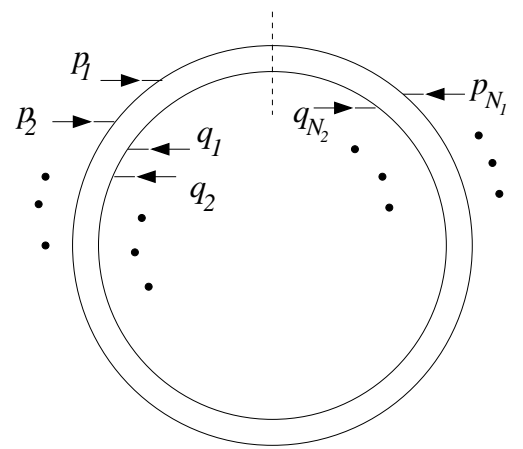

Fig. 1. One-loop Feynman diagram for N-point one-particle-irreducible Green function. The circumference of the vacuum diagram has a length $\mathrm{T}$, while the relative position of each external line from the reference point (dashed vertical) is denoted $\tau_{i}$ 's.

In the limit Eq.4.2), the T-moduli integral is evaluated accurately via the saddle-point approximation. Evidently, $\mathrm{T}=|l| / 2 \mathrm{~m}$ at the saddle point. Then, the integral is evaluated as

$$
\begin{aligned}
\Gamma_{\mathrm{N}}\left[\left\{p_{i}\right\},\left\{q_{j}\right\}\right] & =\hbar\left(2 \pi \frac{|\ell|}{m}\right)^{-\frac{d}{2}}\left(\frac{2 \pi}{m|\ell|}\right)^{1 / 2} e^{-m|\ell|} \\
& \times \sum_{\mathrm{N}_{1}+\mathrm{N}_{2}=\mathrm{N}} C_{\{\mathrm{N}\}}\left\{g^{\mathrm{N}_{1}}|l|^{\mathrm{N}_{1}} J_{\mathrm{N}_{1}}(\ell)\right\}\left\{g^{\mathrm{N}_{2}}|l|^{\mathrm{N}_{2}} J_{\mathrm{N}_{2}}(-\ell)\right\},
\end{aligned}
$$

where $g \equiv-\lambda / 4 m$. The factorized expression permits exponentiation of the double-sum over $\mathrm{N}_{1}, \mathrm{~N}_{2}$. Indeed, summing over $\mathrm{N}=\mathrm{N}_{1}+\mathrm{N}_{2}$, taking carefully into account of the combinatorial factors $C_{\mathrm{N}}$, I find [7, 8

$$
\Gamma=\frac{\hbar}{2} \int \frac{\mathrm{d}^{d} \ell}{(2 \pi)^{d}} W(\ell) \mathcal{K}_{-d}(|\ell|) W(-\ell)
$$


where

$$
\begin{aligned}
\mathcal{K}_{-d}(|\ell|)= & \left(2 \pi \frac{|\ell|}{m}\right)^{-\frac{d}{2}}\left(\frac{2 \pi}{m|\ell|}\right) e^{-m|\ell|} \\
W(\ell)= & \sum_{\mathrm{N}=0}^{\infty} \int \frac{\mathrm{d}^{d} p_{1}}{(2 \pi)^{d}} \cdots \int \frac{\mathrm{d}^{d} p_{\mathrm{N}}}{(2 \pi)^{d}}(2 \pi)^{d} \delta^{d}\left(p_{1}+\cdots+p_{\mathrm{N}}-\ell\right) \\
& \times \frac{1}{\mathrm{~N} !}(-g|\ell|)^{\mathrm{N}}\left[\Phi\left(p_{1}\right) \cdots \Phi\left(p_{\mathrm{N}}\right) \cdot J_{\mathrm{N}}\left(\left\{p_{i}\right\}, \ell\right)\right] .
\end{aligned}
$$

In the last step, I utilized the result of the previous subsection that open Wilson lines are expandable in power-series of $\star_{\mathrm{N}}$ products.

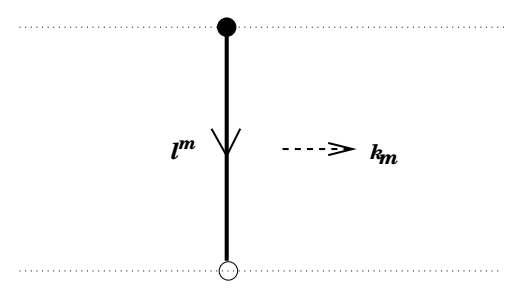

Fig. 2. Spacetime view of open Wilson line propagation. The noncommutativity is turned on the plane spanned by the two vectors, $\ell^{m}$ and $k_{m}$.

The $\mathrm{N}_{1}=\mathrm{N}_{2}=1$ term was computed previously in [16], where infrared singular behavior of the result was interpreted as manifestation of the UV-IR duality. What remained not understood in the work of 16] was understanding reason behind the UV-IR duality. What I have shown above is that this is answerable by summing over N, viz. computing the full effective action, and that the UV-IR duality originates from noncommutative dipole degrees of freedom inherent in any noncommutative field theory. In fact, the one-loop effective action is expressible schematically as

$$
\Gamma_{2}[W]=\frac{1}{2} \operatorname{Tr}_{\mathcal{H}_{\text {dipole }}}\left(\widehat{W} \cdot \mathbf{K}_{2} \cdot \widehat{W}\right), \quad \quad \mathbf{K}_{2}:=\widehat{\mathcal{K}}_{-\frac{d}{2}} .
$$

Here, I have used the Weyl-Moyal correspondence and expressed the open Wilson lines and kernel as operators defined on one-dipole Hilbert space $\mathcal{H}_{\text {dipole. }}$ Remarkably, the action takes strikingly the same form as the quadratic part of matter sector in Witten's cubic open string field theory!

\subsection{Interacting OWLs}

I next compute the nonplanar part of the two-loop effective action and show that it is expressible as cubic interaction of the open Wilson lines. Begin with the two-loop nonplanar contribution to the $N$-point one-particleirreducible Green functions [17]. The Feynman diagram under consideration 
is depicted in Fig.3. Begin with constructing a planar two-loop vacuum diagram 2 constructed by joining three internal propagators via two $\lambda\left[\Phi^{3}\right]_{\star}$ interaction vertices. Denote the internal propagators in double-lines and label them as $a=1,2$, and 3 in Fig.(3). Moduli parameters $\mathrm{T}_{1}, \mathrm{~T}_{2}, \mathrm{~T}_{3}$ refer to the Feynman-Schwinger parameters of the three internal propagators, and range over the moduli space, $\mathcal{M}_{2-\text { loop }}=[0, \infty) \otimes[0, \infty) \otimes[0, \infty)$. We then affix $\mathrm{N}$ external lines (background fields), distributed among the three internal propagators as $\mathrm{N}_{a}(a=1,2,3)$ so that $\left(\mathrm{N}_{1}+\mathrm{N}_{2}+\mathrm{N}_{3}\right)=\mathrm{N}$. Each group of external lines are further classifiable into those affixed from the inner and the outer boundaries. Sum over all possible insertion of the external lines is provided by integration over the moduli parameters $\tau_{i}^{(a)}$, $\mathrm{s}$ over $\left[0, \mathrm{~T}_{a}\right]$, and $\tau_{i j}^{(a)}$ refers to $\left(\tau_{i}^{(a)}-\tau_{j}^{(a)}\right)$.

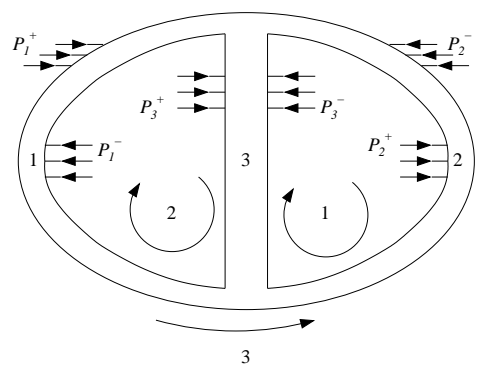

Fig. 3. Two-loop Feynman diagram for N-point Green function. The external lines and the vacuum diagram boundaries are labelled dual each other. The external momenta $p_{i}^{(a)}$, Feynman-Schwinger moduli parameters $\tau_{i}^{(a)}$, and Moyal's phase-factor signs $\nu_{i}^{(a)}$ are ordered from top to bottom. With this ordering convention, the $\tau^{\prime} s$ range over $0<\tau_{\mathrm{N}}<\cdots<\tau_{1}<\mathrm{T}$ for each connected side of the three internal propagators.

Momenta of external lines attached at $a$-th internal propagator are labelled as $\left\{p_{i}^{(a)}\right\}$, where $a=1,2,3$ and $i=1,2, \cdots, \mathrm{N}_{a}$. I introduced total momentum injected on $a$-th internal propagator as:

$$
P_{a}^{ \pm} \equiv \sum_{i=1}^{\mathrm{N}_{a}} \frac{1 \pm \nu_{i}^{(a)}}{2} p_{i}^{(a)} \quad \text { and } \quad P_{a} \equiv P_{a}^{+}+P_{a}^{-},
$$

where \pm refers to the inner and the outer boundaries, respectively, and $\nu_{i}^{(a)}$ takes \pm 1 depending on whether the insertion is made from the 'left' or the

\footnotetext{
${ }^{2}$ At two loop and beyond, vacuum diagrams are classifiable into a planar diagram and the rest, nonplanar diagrams. If the number of twist insertion is zero, the vacuum diagram is referred as planar. All other vacuum diagrams, with at least one insertion of the twist, are nonplanar ones. At one loop, by default, the vacuum diagram is planar.
} 
'right' side. I have also introduced the total momentum inserted on each worldsheet boundary via $k_{a+2}=P_{a}^{+}+P_{a+1}^{-}$. Again, taking the limit Eq. (4.2) and consuming a lengthy computation, I have obtained:

$\Gamma_{\mathrm{N}}\left(\left\{p_{a}^{(a)}\right\}\right)=\frac{\hbar^{2} \lambda^{2}}{24}\left(-\frac{\lambda}{2}\right)^{\mathrm{N}} \sum_{\left\{\mathrm{N}_{a}\right\}=0}^{\mathrm{N}} \sum_{\{\nu\}}(2 \pi)^{d} \delta\left(\sum_{a=1}^{3} \sum_{n=1}^{\mathrm{N}_{a}} p_{n}^{(a)}\right) C_{\{\mathrm{N}\}} \Gamma_{\nu}^{\left(\left\{\mathrm{N}_{a}\right\}\right)}$.

Here, $C_{\{\mathrm{N}\}}$ denotes a combinatoric factor, and

$$
\begin{aligned}
\Gamma_{\sigma, \nu}^{\left(\mathrm{N}_{1}, \mathrm{~N}_{2}, \mathrm{~N}_{3}\right)} & =\int_{0}^{\infty} \frac{\mathrm{dT}_{1} \mathrm{dT}_{2} \mathrm{dT}_{3}}{(4 \pi)^{d}} e^{F\left(\mathrm{~T}_{1}, \mathrm{~T}_{2}, \mathrm{~T}_{3}\right)} \Delta^{\frac{d}{2}}(\mathrm{~T})\left(\prod_{a=1}^{3} \int_{0}^{\mathrm{T}_{a}} \prod_{i=1}^{\mathrm{N}_{a}} \mathrm{~d} \tau_{i}^{(a)}\right) \\
& \times \prod_{a=1}^{3}\left(\sum_{\mathrm{N}_{a}^{+}} \frac{\mathrm{T}_{a}^{\mathrm{N}_{a}^{+}}}{\mathrm{N}_{a}^{+} !} J_{\mathrm{N}_{a}^{+}}\left(+\alpha_{a}\right)\right)\left(\sum_{\mathrm{N}_{a}^{-}} \frac{\mathrm{T}_{a}^{-}}{\mathrm{N}_{a}^{-} !} J_{\mathrm{N}_{a}^{-}}\left(-\alpha_{a}\right)\right)
\end{aligned}
$$

in which

$$
\begin{gathered}
\exp F\left(\mathrm{~T}_{1}, \mathrm{~T}_{2}, \mathrm{~T}_{3}\right)=\exp \left[-m^{2}\left(\mathrm{~T}_{1}+\mathrm{T}_{2}+\mathrm{T}_{3}\right)-\frac{\Delta}{4}\left(\mathrm{~T}_{1} \ell_{1}^{2}+T_{2} \ell_{2}^{2}+\mathrm{T}_{3} \ell_{3}^{2}\right)\right] \\
\Delta^{-1}(\mathrm{~T}):=\mathrm{T}_{1} \mathrm{~T}_{2}+\mathrm{T}_{2} \mathrm{~T}_{3}+\mathrm{T}_{3} \mathrm{~T}_{1} \\
\widehat{J}_{\left(\mathrm{N}_{a}^{+}, \mathrm{N}_{a+1}^{-}\right)}\left(-\alpha_{a}, \alpha_{a+1}\right)=\exp \left[\frac{i}{2} t_{a} t_{a+1} k_{a} \wedge k_{a+1}\right] \widetilde{J}_{\mathrm{N}_{a}^{+}} \widetilde{J}_{\mathrm{N}_{a+1}^{-}}
\end{gathered}
$$

and $\widetilde{J}$ 's are precisely the same as the one-loop $\star_{\mathrm{N}}$ kernel. I have also introduced the following shorthand notations:

$$
\begin{aligned}
t_{a} & =\sqrt{\Delta} \mathrm{T}_{a}, \quad\left(t_{1} t_{2}+t_{2} t_{3}+t_{3} t_{1}=1\right) \\
\alpha_{1} & =t_{1}\left(t_{2} \ell_{2}-t_{3} \ell_{3}\right), \quad \alpha_{2}=t_{2}\left(t_{3} \ell_{3}-t_{1} \ell_{1}\right), \quad \alpha_{3}=t_{3}\left(t_{1} \ell_{1}-t_{2} \ell_{2}\right) .
\end{aligned}
$$

Geometrically, for any given nonnegative values of $\left\{t_{a}\right\},\left\{\alpha_{a}\right\}$ split the triangle formed by $\left\{\ell_{a}\right\}$ into three pieces, viz., $\ell_{1}=\alpha_{3}-\alpha_{2}, \ell_{2}=\alpha_{1}-\alpha_{3}$, $\ell_{3}=\alpha_{2}-\alpha_{1}$.

The T-moduli integrals are computable by saddle-point conditions. $\partial F /$ $\partial \mathrm{T}_{a}=0$ yields

$$
\Delta^{-1}=\frac{L^{2}}{4 m^{2}} \quad \text { and } \quad L \equiv\left|t_{1} l_{1}-t_{2} l_{2}\right|=\left|t_{2} l_{2}-t_{3} l_{3}\right|=\mid t_{3} l_{3}-t_{1} l_{1} .
$$

They determine the "size" of the moduli and their relative "angles". Geometrically, the condition Eq.(4.4) demands that the angle between a pair of $\alpha$ 's is $2 \pi / 3$. Moreover, the value of $F\left(\left\{\mathrm{~T}_{a}\right\}\right)$ at the saddle point also has 
a simple geometric description: $F($ saddle $)=-m\left(\left|\alpha_{1}\right|+\left|\alpha_{2}\right|+\left|\alpha_{3}\right|\right)_{\text {saddle }}$. The crucial point is that, at the saddle point, I now have

$$
\mathrm{T}_{a}=\Delta^{-1 / 2} t_{a}=\frac{L}{2 m} t_{a}=\frac{\left|\alpha_{a}\right|}{2 m},
$$

Plugging Eq.(4.5) into Eq.(4.3), each factor sums up to an open Wilson line

$$
\sum_{\mathrm{N}_{a}^{+}}\left(-\frac{\lambda}{2}\right)^{\mathrm{N}_{a}^{+}} \frac{\mathrm{T}_{a}^{\mathrm{N}_{a}^{+}}}{\mathrm{N}_{a}^{+} !} J_{\mathrm{N}_{a}^{+}}\left(\alpha_{a}\right)=\sum_{\mathrm{N}_{a}^{+}}\left(-\frac{\lambda}{4 m}\left|\alpha_{a}\right|\right)^{\mathrm{N}_{a}^{+}} J_{\mathrm{N}_{a}^{+}}\left(\alpha_{a}\right),
$$

just like the one-loop case, except that the Wilson line contour is now 'snapped'! I finally obtain the two-loop effective action as 18

$$
\begin{aligned}
\Gamma[\ell] & =\frac{1}{3} \lambda^{2} \hbar^{2} \int \frac{\mathrm{d}^{d} k_{1}}{(2 \pi)^{d}} \cdots \frac{\mathrm{d}^{d} k_{3}}{(2 \pi)^{d}} \delta^{(d)}\left(k_{1}+k_{2}+k_{3}\right)\left(\frac{2 m}{L}\right)^{d-3}\left(\frac{\delta \mathrm{T}}{\mathrm{T}}\right)^{3} \\
& \times \exp \left[-m\left(\left|\alpha_{1}\right|+\left|\alpha_{2}\right|+\left|\alpha_{3}\right|\right)\right] \exp \left(-\frac{i}{2} \sum_{a=1}^{3} \alpha_{a} \wedge \alpha_{a+1}\right) \\
& \times\left[\widehat{W}\left(\alpha_{1},-\alpha_{2}\right)\right]\left[\widehat{W}\left(\alpha_{2},-\alpha_{3}\right)\right]\left[\widehat{W}\left(\alpha_{3},-\alpha_{1}\right)\right] .
\end{aligned}
$$

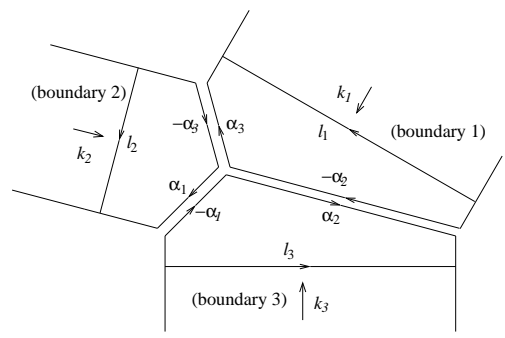

Fig. 4. Spacetime view of interaction among three open Wilson lines. At asymptotic region, the dipoles are described by straight open Wilson lines, while, at interaction region, the dipoles interact locally pairwise by 'snapping' the open Wilson lines.

Again, the two-loop effective action is expressible schematically as:

$$
\Gamma_{3}[W]=\frac{\lambda_{\mathrm{c}}}{3} \operatorname{Tr}_{\mathcal{H}_{\text {dipole }}} \mathbf{K}_{3}(\widehat{W} \widehat{\star} \widehat{W} \widehat{\star} \widehat{W}) \quad \text { where } \quad \lambda_{\mathrm{c}}=(\lambda / 2)^{2},
$$

where $\mathbf{K}_{3}$ represents a weight-factor over one-dipole Hilbert space $\mathcal{H}_{\text {dipole }}$, and the $\widehat{x}$-product refers to a newly emergent noncommutativity in the algebra of open Wilson lines. I note that the two-loop effective action, once expressed in terms of the snapped open Wilson lines, is remarkably similar 
to 'half-string' picture of the cubic interaction in Witten's open string field theory, again restricted to the matter sector, with a minor difference that location of the triple interaction point is determined dynamically by the energy-momentum carried by the asymptotic dipoles. Note also that 'softdilaton theorem' is obeyed - the interaction strength $\lambda_{\mathrm{c}}$ is proportional to square of the $\Phi$-field coupling parameter, $\lambda$.

\section{Closed Strings out of Open Strings}

The last conjecture of mine concerns extrapolation of the observations made in noncommutative field theories to string theories: closed strings are created/annihilated by 'open Wilson lines' made out of open string fields [20].

The starting point would be that, at level-zero truncation and in the background of nonzero two-form potential $B$, Witten's cubic open string field theory is approximated by a noncommutative field theory of the open string tachyon with a cubic interaction. Expand the tachyon potential around local minimum. The resulting theory reduces precisely to the $\lambda\left[\Phi^{3}\right]_{\star^{-}}$ theory I have discussed at length already, but, quite importantly, with the mass-squared $m^{2} \rightarrow \infty$. According to Sen's conjecture, excitation around the tachyon potential minimum ought to correspond to that of a closed string only and none of the open string. A point to be explored is "can one understand Sen's conjecture even with zero-level truncation, viz. a noncommutative $\lambda\left[\Phi^{3}\right]_{\star}$ scalar field theory? I now claim that the answer to this question is affirmatively yes.

\subsection{Open String as Miniature Dipole}

The starting point is the observation that open strings in a constant Bfield backgrounds are noncommutative dipoles, albeit of miniature size. In conformal gauge, an open string worldsheet action is

$S_{\text {open }}=\frac{1}{2 \ell_{\mathrm{st}}^{2}} \int_{\Sigma}\left[\left(G_{m n} \delta^{\alpha \beta}+B_{m n} \epsilon^{\alpha \beta}\right) \partial_{\alpha} X^{m} \partial_{\beta} X^{n}\right]+\int_{\partial_{\mathrm{L} \Sigma}-\partial_{\mathrm{R}} \Sigma} A_{m}(X) \dot{X}^{m}$,

where $\partial_{\mathrm{L}, \mathrm{R}} \Sigma$ refers to left or right boundary of the worldsheet $\Sigma$. The $B$-field is locally exact, and can be gauged away via $\mathrm{U}(1)$ transformation: $B_{2} \rightarrow B_{2}+d \Lambda_{1}, A_{1} \rightarrow A_{1}-\ell_{\mathrm{st}}^{-2} \Lambda_{1}$. This results in

$$
S_{\mathrm{open}}=\frac{1}{2 \ell_{\mathrm{st}}^{2}} \int_{\Sigma} G_{m n}\left(\dot{X}^{m} \dot{X}^{n}-X^{\prime m} X^{\prime n}\right)+\int_{\partial_{\mathrm{L}} \Sigma-\partial_{\mathrm{R}} \Sigma} A_{m}(X) \dot{X}^{m} .
$$

I now latticize the open string by two points, separated by worldsheet length $2 \ell_{\text {st }}$. Let $X(0, \tau)=X_{\mathrm{L}}(\tau)$ and $X\left(2 \ell_{\mathrm{st}}, \tau\right)=X_{\mathrm{R}}(\tau)$. The open string action 
is then approximatable as

$$
\begin{aligned}
S_{\text {open }} & \rightarrow \int d \tau\left[\frac{1}{2} m\left(\dot{X}_{\mathrm{L}}^{2}+\dot{X}_{\mathrm{R}}^{2}\right)-\frac{1}{2} m \omega^{2}\left(X_{\mathrm{L}}-X_{\mathrm{R}}\right)^{2}\right] \\
& +\int d \tau\left[q B_{m n}\left(\dot{X}_{\mathrm{L}}^{m} X_{\mathrm{L}}^{n}-\dot{X}_{\mathrm{R}}^{m} X_{\mathrm{R}}^{n}\right)\right]
\end{aligned}
$$

where $m=\omega=1 / \ell_{\mathrm{st}}$ and $q=1 / \ell_{\mathrm{st}}^{2}$. Alternatively, in terms of center-ofmass and relative coordinates, $\mathbf{R}=\left(X_{\mathrm{L}}+X_{\mathrm{R}}\right) / 2$ and $\ell=\left(X_{\mathrm{L}}-X_{\mathrm{R}}\right)$,

$$
S_{\text {open }}=\int d \tau\left[\frac{1}{2} M \dot{\mathbf{R}}^{2}+\frac{1}{2} \mu \dot{\ell}^{2}-\frac{1}{2} \mu \omega^{2} \ell^{2}+\frac{q}{2} B_{m n}\left(\dot{\mathbf{R}}^{m} \ell^{n}-\dot{\ell}^{m} \mathbf{R}^{n}\right)\right],
$$

where $M=2 m, \mu=m / 2$. In either form, it shows that the two-point latticized open string is literally identical to the Mott exciton or the noncommutative dipole. From the action, I also infer the boundary conditions:

$$
\begin{aligned}
m \omega^{2}\left(X_{\mathrm{L}}-X_{\mathrm{R}}\right)_{m}+q F_{m n}\left(X_{\mathrm{L}}\right) \dot{X}_{\mathrm{L}}^{n} & =0 \\
m \omega^{2}\left(X_{\mathrm{R}}-X_{\mathrm{L}}\right)_{m}-q F_{m n}\left(X_{\mathrm{R}}\right) \dot{X}_{\mathrm{R}}^{n} & =0 .
\end{aligned}
$$

I now quantize the open string, viz. first-quantize the two point particles inside the dipole. In doing so, the boundary conditions Eq.(5.1) needs to be imposed as constraints. The dipole coordinates then obey exactly the same commutation relations as those obeyed by the Mott exciton:

$$
\left[X_{\mathrm{L}}^{m}, X_{\mathrm{L}}^{n}\right]=+i \theta^{m n}, \quad\left[X_{\mathrm{R}}^{m}, X_{\mathrm{R}}^{n}\right]=-i \theta^{m n}, \quad\left[X_{\mathrm{L}}^{m}, X_{\mathrm{R}}^{n}\right]=0
$$

or, equivalently, in the notation adopted for the Mott exciton,

$$
\left[\mathbf{R}^{m}, \ell^{n}\right]=i \theta^{m n}, \quad\left[\mathbf{R}^{m}, \mathbf{R}^{n}\right]=0=\left[\ell^{m}, \ell^{n}\right] .
$$

These commutation relations indicate that the open string is a noncommutative dipole, obeying the dipole relation: one-dipole Hilbert space is simply a tensor product of two one-particle Hilbert spaces: $\mathcal{H}_{\text {dipole }}=\mathcal{H}_{\mathrm{L}} \otimes \mathcal{H}_{\mathrm{R}}$. In light of the latticization employed, one-dipole Hilbert space should be identified with first-quantized string Hilbert space.

\subsection{Witten's $\star_{\mathrm{w}}$-product is Moyal's $\star_{\mathrm{m}}$-product}

Utilizing the dipole picture of latticized open string, I now argue that the $\star$-product defining Witten's open string theory is identifiable as Moyal's $\star$-product. Actually, in the absence of constant B-field background, the isomorphism holds for string oscillator modes, but not for zero mode. Once B-field is turned on, the isomorphism hold exactly including the zero mode. This is the reason why we started with Witten's open string field theory in B-field background. 
Schematically, Witten's $\star_{\mathrm{w}}$ product is defined on string Hilbert space $\mathcal{H}_{\mathrm{L}} \otimes \mathcal{H}_{\mathrm{R}}$ by

$$
\left(\left|x_{\mathrm{L}}\right\rangle \otimes\left|x_{\mathrm{R}}\right\rangle\right) \star_{\mathrm{w}}\left(\left|y_{\mathrm{L}}\right\rangle \otimes\left|y_{\mathrm{R}}\right\rangle\right) \longrightarrow\left\langle x_{\mathrm{R}} \mid y_{\mathrm{L}}\right\rangle\left(\left|x_{\mathrm{L}}\right\rangle \otimes\left|y_{\mathrm{R}}\right\rangle\right) .
$$

Because of the dipole relation, I advocate the viewpoint treating the dipole configuration space $\left(X_{\mathrm{L}}, X_{\mathrm{R}}\right)$ as a one-particle phase-space $(\mathbf{R}, \mathbf{P})$ associated with dipole's center-of-mass, where $\mathbf{P}=\theta^{-1} \cdot \ell$. Then, via Weyl-Moyal correspondence, Moyal's $\star_{\mathrm{m}}$ product in $(\mathbf{R}, \mathbf{P})$ space ought to be equivalent to matrix product in $\left(X_{\mathrm{L}}, X_{\mathrm{R}}\right)$ space. It then follows that Moyal's $\star_{\mathrm{m}}$ product equals to Fourier transform of Witten's $\star_{\mathrm{w}}$ product.

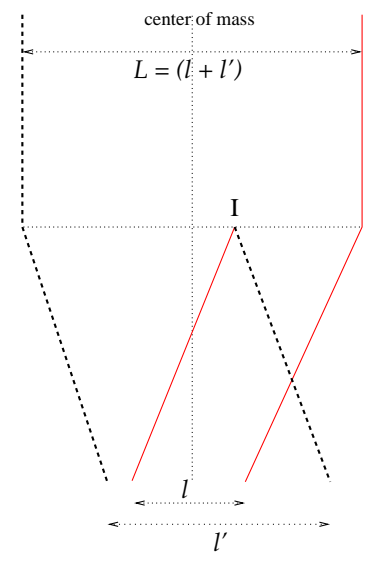

Fig. 5. Interaction of two miniature dipoles in support of equivalence between Moyal's $\star_{\mathrm{m}}$ product and Witten's $\star_{\mathrm{w}}$ product. Interaction point $I$ can be anywhere and should be summed over.

Explicitly, start with Moyal's $\star_{\mathrm{m}}$-product in $(\mathbf{R}, \mathbf{P})$ space:

$$
\left[A \star_{\mathrm{m}} B\right](\mathbf{R}, \mathbf{P})=A(\mathbf{R}, \mathbf{P}) \exp \left[\frac{i}{2}\left(\overleftarrow{\partial_{\mathbf{R}}} \cdot \overrightarrow{\partial_{\mathbf{P}}}-\overleftarrow{\partial_{\mathbf{P}}} \cdot \overrightarrow{\partial_{\mathbf{R}}}\right)\right] B(\mathbf{R}, \mathbf{P})
$$

I next "Fourier transform" with respect to $\mathbf{P}$ and express all in terms of dipole's relative distance $\ell$ 's:

$$
A(\mathbf{R}, \mathbf{P})=\int d \ell e^{-i \mathbf{P} \cdot \ell} \widetilde{A}(\mathbf{R}, \ell) \quad \text { and } \quad B(\mathbf{R}, \mathbf{P})=\int d \ell e^{-i \mathbf{P} \cdot \ell} \widetilde{B}(\mathbf{R}, \ell) .
$$

Substituting so, Eq.(5.3) is considerably simplified after a change of variables: $L=\left(\ell+\ell^{\prime}\right), L^{\prime}=\left(\ell^{\prime}-\ell\right)$. Fourier transforming back the whole expression in Eq. 5.3. with respect to $\mathbf{P}$, I obtain [19]:

$\left[A \widetilde{\star_{\mathrm{m}}} B\right](\mathbf{R}, L)=\int_{-\infty}^{+\infty} \frac{d L^{\prime}}{2} \widetilde{A}\left(\mathbf{R}+L / 2, \mathbf{R}+L^{\prime} / 2\right) \widetilde{B}\left(\mathbf{R}+L / 2, \mathbf{R}-L^{\prime} / 2\right) .(5.4)$ 
The emerging picture is that a miniature dipole $\widetilde{A}$ at center $\mathbf{R}$ and of length $\ell$ and another $\widetilde{B}$ at center $\mathbf{R}$ and of length $\ell^{\prime}$ come into contact. When interacting, $\widetilde{A}, \widetilde{B}$ shift their centers to $\mathbf{R}+\ell^{\prime}$ and $\mathbf{R}-\ell$, respectively. The final dipole $A \star_{\mathrm{m}} B$ is then centered at $\mathbf{R}$ and of length $L=\left(\ell+\ell^{\prime}\right)$. See Fig. Evidently, the dipole interaction Eq. (5.4) defined via Moyal's $\star_{\mathrm{m}}$ product yields is algebraically equivalent to the string field interaction Eq.(5.2) defined via Witten's $\star_{\mathrm{w}}$ product.

\subsection{Closed Strings as OWLs}

Recall that I have identified the scalar field $\Phi$ with the level-zero mode of the open string field. If I focus on low-energy and low-momentum excitation below a fixed cutoff, $p^{2} \leq \Lambda^{2}$, as $m^{2} \rightarrow \infty$, excitation of the $\Phi$-quanta is entirely suppressed. This is clearly counterpart of half of Sen's conjecture: 'around the tachyon potential minimum, there is no open string excitation'. The regime $\Lambda^{2} \ll m^{2}$ is also of considerable relevance to the effective action computation in $\lambda\left[\Phi^{3}\right]_{\star}$-theory, which I have not discussed at all so far. The point is that, in addition to the nonplanar diagram contribution, there also exists the planar diagram contribution to the effective action. The planar part is actually sensitive to the UV cutoff. If I identify the UV cutoff with the fixed cutoff $\Lambda$ and take the conventional limit $m^{2} \ll \Lambda^{2}$, the planar part of the effective action yields a sort of Coleman-Weinberg type potential (plus derivative corrections) - viz. exponentiation of the scalar field $\Phi$ takes place. On the other hand, if I take the opposite limit, $\Lambda^{2} \ll m^{2}$, I have found that the planar diagram contribution turns remarkably into the same functional form as the nonplanar diagram contribution, except that (some of) the open Wilson lines carry nearly zero momentum. The point is that, even for planar diagrams, the scalar field $\Phi$ is exponentiated into open Wilson lines, albeit miniature ones, provided the cutoff condition obeys $\Lambda^{2} \ll m^{2}$. While quite bizzare from the standard quantum field theory viewpoint, to one's delight, this cutoff condition is precisely what is dictated by Witten's open string field theory!

The other half of Sen's conjecture - closed string out of open string tachyon vacuum - is then readily inferred from the results of previous sections. The open Wilson line formed out of the tachyon field $\Phi$ is precisely the interpolating operator creating and annihilating a closed string. The fact that open Wilson lines are Moyal formulation counterpart of the Wilson loop in Weyl formulation adds an another supporting evidence for this claim [20]. There is one peculiar aspect, though. First of all, the spacetime structure of the open Wilson lines is literally open, viz. the two ends are situated at distinct points in the target space. Moreover, the cubic interaction of the open Wilson lines, Eq.(4.6), involves newly emergent $\widehat{\star}$-product. As both are the aspects inherently associated with traditional open strings, one might feel suspicious to my conjecture of identifying the open Wilson 
lines as closed strings. I claim that a resolution can be drawn from the wellknown fact that closed string is formed by joining two ends of open string(s). In the absence of the two-form potential, $B_{m n}=0$, size of the open string is characteristically of string scale, and is too small to be probed by the levelzero truncated tachyon field. If the two-form potential is nonzero, $B_{m n} \neq 0$, the open string is polarized to a size much bigger than the string scale, and behaves essentially like a rigid rod. Because of that, joining and splitting of the two end of open string(s) would never form a closed string. In other words, open Wilson lines are precisely what the open strings can do the best for forming closed strings out of themselves! Reverting the logic, utility of turning on the B-field and hence noncommutativity for the open string is to render closed strings as much the same as open strings. That the open Wilson lines interaction is governed by a newly emergent $\widehat{\star}$-product (see Eq.(4.6) would then constitute a nontrivial prediction of the conjectures I put forward.

\section{acknowledgement}

This work was supported in part by BK-21 Initiative in Physics (SNU Project 2), KOSEF Interdisciplinary Research Grant 98-07-02-07-01-5 and KOSEF Leading Scientist Program.

\section{References}

[1] N. Read, Semicond. Sci. Technol. 9 (1994) 1859; Surf. Sci. 361 (1996) 7; V. Pasquier, (unpublished); R. Shankar and G. Murthy, Phys. Rev. Lett. 79 (1997) 4437; D.-H. Lee, Phys. Rev. Lett. 80 (1998) 4745; V. Pasquier and F.D.M. Haldane, Nucl. Phys. B516[FS] (1998) 719; A. Sterm, B.I. Halperin, F. von Oppen and S. Simon, Phys. Rev. B59 (1999) 12547.

[2] D. Bigatti and L. Susskind, Phys. Rev. D66 (2000) 066004, hep-th/9908056.

[3] S.-J. Rey and R. von Unge, Phys. Lett. B499 (2001) 215, hep-th/0007089.

[4] S.R. Das and S.-J. Rey, Nucl. Phys. B590 (2000) 453, hep-th/0008042.

[5] D.J. Gross. A. Hashimoto and N. Itzhaki, Adv. Theor. Math. Phys. 4 (2000) 893, hep-th/0008075.

[6] N._shibashi.S. Iso, H. Kawai and Y. Kitazawa, Nucl. Phys. B 573 (2000) 573, hep-th/9910004.

[7] Y. Kiem.S.-J. Rey, H.-T. Sato, and J.-T. Yee, Phys. Rev. D65 (2002) 026002, hep-th/0106121.

[8] Y. Kiem. S.-J.j Rey, H. Sato, and J.-T. Yee, Eur. Phys. J. C22 (2002) 757, hep-th/0107106.

[9] Y. Kiem, S.-J. Rey, H.-T. Sato, and J.-T. Yee, Eur. Phys. J. C22 (2002) 781.

[10] G. Parisi, Phys. Lett. B112 (1982) 463.

[11] T. Mehen and M.B. Wise, J. High-Energy Phys. 0012 (2000) 008, hep-th/0010204;

[12] H. Liu, hep-th/0011125.

[13] M. R. Garousi, Nucl. Phys. B579 (2000) 209, hep-th/9909214

[14] H. Liu and J. Michelson, hep-th/0008205. 
[15] H. Liu, hep-th/0011125.

[16] S. Minwalla, M. Van Raamsdonk and N. Seiberg, JHEP 0002 (2000) 020; M. Van Raamsdonk and N. Seiberg, JHEP 0003 (2000) 035.

[17] Y. Kiem, S.-S. Kim, S.-J. Rey, and H.-T. Sato, hep-th/0110066.

[18] Y. Kiem. S. Lee, S.-J. Rey and H.-T. Sato, Phys. Rev. D65 (2002) 046003, hep-th/0110215.

[19] S.-J. Rey, unpublished note (2001); I. Bars and S.-J. Rey, Phys. Rev. D64, (2001) 046005, hep-th/0104135.

[20] S.-J. Rey, Half-string, Noncommutative Geometry and String Field Theory, to appear. 